EPJ Web of Conferences 49, 15002 (2013)

DOI: $10.1051 /$ epjconf/20134915002

(C) Owned by the authors, published by EDP Sciences, 2013

\title{
Search For New Physics at BABAR
}

\author{
Romulus Godang University of South Alabama,a \\ ${ }^{1}$ Department of Physics \\ University of South Alabama \\ ILB 115, 307 University Blvd., N.
}

SLAC-PUB-15354

USA-HEP-2013-02

UMS-HEP-2013-02

\begin{abstract}
Using a full BABAR data sample of $426 \mathrm{fb}^{-1}$, we present improved measurements of the ratio $\mathcal{R}\left(D^{(*)}\right)=\mathcal{B}\left(\bar{B} \rightarrow D^{(*)} \tau^{-} \bar{v}_{\tau}\right) / \mathcal{B}\left(\bar{B} \rightarrow D^{(*)} \ell_{\ell}^{-} \bar{v}_{\ell}\right)$, where $\ell$ is either electron or muon. We measure $\mathcal{R}(D)=$ $0.440 \pm 0.058 \pm 0.042$ and $\mathcal{R}\left(D^{*}\right)=0.332 \pm 0.024 \pm 0.018$. These ratios exceed the Standard Model predictions by $2.0 \sigma$ and $2.7 \sigma$, respectively. The results disagree with the Standard Model predictions at the level of $3.4 \sigma$. The ratios are sensitive to new physics contributions in the form of a charged Higgs boson. However, the access cannot be explained by a charged Higgs boson in the type II two-Higgs-doublet model.
\end{abstract}

\section{INTRODUCTION}

The semileptonic physics in $B$ meson sector played a prominent role in investigating of new physics effect at low-energy region. Semileptonic transitions are the simplest process in $B$ mesons decay. In the Standard Model (SM), the heavy $b$ quark decays to either a $c$ or an $u$ quark and the virtual $W$ boson [1-3]. Experimentally, semileptonic decays have the advantage of large branching fraction and are used to determine the weak couplings, the Cabibbo-Kobayashi-Maskawa (CKM) matrix elements, $\left|V_{c b}\right|$ and $\left|V_{u b}\right|[4]$.

The parton level diagram of $\bar{B} \rightarrow D^{(*)} \tau^{-} \bar{v}_{\tau}$ decays where $D^{(*)}$ refers to either a $D$ or a $D^{*}$ meson is shown in Fig. 1. The decay $\bar{B} \rightarrow D^{(*)} \tau^{-} \bar{v}_{\tau}$ is sensitive to charged Higgs contribution at the tree level. The three-body de-

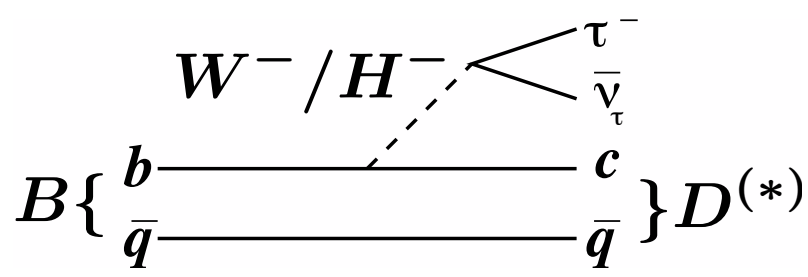

Figure 1. The parton level diagram for $\bar{B} \rightarrow D^{(*)} \tau^{-} \bar{\nu}_{\tau}$ decays

cay $\bar{B} \rightarrow D^{(*)} \tau^{-} \bar{v}_{\tau}$ permits the study of decay distribution which discriminate between $W^{-}$and $H^{-}$exchange $[5,6]$.

The decay of $\bar{B} \rightarrow D^{(*)} \tau^{-} \bar{v}_{\tau}$ with $\tau$ lepton in the final state offer possibilities of significant new physics contribu-

\footnotetext{
${ }^{\mathrm{a}}$ This work was supported by the U.S. Department of Energy under grant No. DE-FG02-96ER-40970 e-mail: godang@usouthal.edu
}

tions that is not present in the process where light lepton such as electron and muon in the final state. The study of $\bar{B} \rightarrow D^{(*)} \tau^{-} \bar{v}_{\tau}$ have already shown the new physics contributions can be over-constrained [7-10]. The existing studies of the $\bar{B} \rightarrow D^{(*)} \tau^{-} \bar{v}_{\tau}$ based on two-Higgs-doublet model (2HDM) predict a substantial impact on the ratio $\mathcal{R}\left(D^{(*)}\right)$ and $\mathcal{R}(D)[7,8,10,12]$.

\section{The BABAR DETECTOR AND DATA SET}

The BABAR detector was operated at the PEP-II asymmetric-energy storage rings at the SLAC National Accelerator Laboratory. The data used in this analysis were collected with the BABAR detector. We analyze data recorded with the BABAR detector at a center of mass energy of $10.58 \mathrm{GeV}$. The data sample consist of an integrated luminosity of $426 \mathrm{fb}^{-1}$, corresponding to 471 $\times 10^{6} B \bar{B}$ pairs. An additional sample of $40 \mathrm{fb}^{-1}$, taken at energy $40 \mathrm{MeV}$ below the $\Upsilon(4 S)$ resonance. This additional sample of data is used to study the continuum background from the decays of $e^{+} e^{-} \rightarrow q \bar{q}(\gamma)$ pairs where $q$ can be $u, d, s, c, \tau$.

A detail description of the BABAR detector is presented elsewhere [11]. The momenta of the charged particles are measured in a tracking system consisting of a 5-layer double sided silicon vertex tracker (SVT) and a 40-layer drift chamber (DCH). The SVT and DCH operate within a $1.5 \mathrm{~T}$ solenoid field and have a combined solid angle coverage in the center of mass frame of $90.5 \%$. A detector of internally reflected Cerenkov radiation (DIRC) is used for charged particle identifications of pions, kaons, and protons with likelihood ratios calculated from $d E / d x$ 
measurements in the SVT and DCH. Photons and longlived neutral hadrons are detected and their energies are measured in a CsI(Tl) electromagnetic calorimeter (EMC). For electrons, energy lost due to bremsstrahlung is recovered from deposits in the EMC.

\section{ANALYSIS}

In this analysis, instead of measuring the absolute branching fraction of $\bar{B} \rightarrow D^{(*)} \tau^{-} \bar{v}_{\tau}$, we measure the ratios

$$
\mathcal{R}\left(D^{(*)}\right)=\frac{\mathcal{B}\left(\bar{B} \rightarrow D^{(*)} \tau^{-} \bar{v}_{\tau}\right)}{\mathcal{B}\left(\bar{B} \rightarrow D^{(*)} \ell^{-} \bar{v}_{\ell}\right)}
$$

where $\ell$ is either electron or muon. In the standard model (SM), the relative rate $\mathcal{R}\left(D^{(*)}\right)$ have less than $6 \%$ uncertainty [12]. In the decay of $\bar{B} \rightarrow D^{(*)} \tau^{-} \bar{v}_{\tau}$, we construct the $\tau$ lepton only from the purely lepton decays: $\tau^{-} \rightarrow e^{-} \bar{\mu}_{e} v_{\tau}$ and $\tau^{-} \rightarrow e^{-} \bar{v}_{\mu} \nu_{\tau}$ so that the signal events $\left(\bar{B} \rightarrow D^{(*)} \tau^{-} \bar{v}_{\tau}\right)$ and the normalization events $\left(\bar{B} \rightarrow D^{(*)} \ell^{-} \bar{v}_{\ell}\right)$ are identified by the same particles in the final state. When taking the ratio of $\mathcal{R}\left(D^{(*)}\right)$, the various sources of uncertainties will be canceled and reduced.

We reconstruct candidate events produced in $\Upsilon(4 S) \rightarrow$ $B \bar{B}$ decays by selecting the hadron decay of one of the $B$ meson $\left(B_{\text {tag }}\right)$. The other candidate events are reconstructed semileptonically, specially a charm meson (either charged or neutral $D$ or $D^{*}$ ) and a charged lepton (either $e$ or $\mu$ ). The signal events $\left(\bar{B} \rightarrow D^{(*)} \tau^{-} \bar{v}_{\tau}\right)$ and the normalization events $\left(\bar{B} \rightarrow D^{(*)} \ell^{-} \bar{v}_{\ell}\right)$ are extracted using unbinned maximum-likelihood fit to the two-dimensional distributions of the invariant mass of the undetected particles. Basically it is the invariant mass of the neutrinos.

$$
m_{m i s s}^{2}=p_{m i s s}^{2}=\left(p_{e^{+} e^{-}}-p_{B_{\text {tag }}}-p_{D^{(*)}}-p_{\ell}\right)^{2}
$$

where $p$ is the four-momenta of the colliding beams, $B_{\text {tag }}$, $D^{(*)}$ and charged lepton, respectively. The lepton threemomentum in the $B$ rest frame is denoted by $p_{\ell}^{*}$. The distribution of the lepton three-momentum of the signal events is softer than the distribution of the lepton threemomentum of the normalization events because the observed lepton in the signal events is a secondary particle originated from the $\tau$ decay, $\tau^{-} \rightarrow \ell^{-} \bar{v}_{\ell} \nu_{\tau}$.

If all particles are properly reconstructed the invariant mass of the undetected particles $\left(m_{m i s s}^{2}\right)$ with a single missing neutrino peaks at zero, whereas the signal events which have three missing neutrinos have a wide $m_{\text {miss }}^{2}$ distribution that extends from $-1 \mathrm{GeV}^{2}$ to $10 \mathrm{GeV}^{2}$. The two observable kinematic variables are used to select the $B_{\text {tag }}$ candidates:

$$
m_{E S}=\sqrt{E_{\text {beam }}^{2}-p_{\text {tag }}^{2}}
$$

and

$$
\Delta E=E_{\text {tag }}-E_{\text {beam }}
$$

where the $p_{\text {tag }}$ and $E_{\text {tag }}$ refer to the center-of-mass momentum and energy of the $B_{\text {tag }}$. $E_{\text {beam }}$ is the center-of-mass of a single beam particle. If the $B$ decays are correctly reconstructed, the distribution of the $m_{E S}$ is centered at the
$B$ meson mass with a resolution of $2.5 \mathrm{MeV}$. The distribution of $\Delta E$ is centered at zero with a resolution of $18 \mathrm{MeV}$. In this analysis we required $m_{E S}>5.27 \mathrm{GeV}$ and $|\Delta E|<$ $0.072 \mathrm{GeV}$.

The main background contributions to the signal events are the following:

- The decay of $\bar{B} \rightarrow D^{* *}\left(\tau^{-} / \ell^{-}\right) \bar{v}_{\ell}$ where $D^{* *}$ mesons refer to the charm resonances heavier than the $D^{*}$ meson such as $D_{0}^{*}, D_{1}, D_{1}^{\prime}$, and $D_{2}^{*}$ orbital excitations of the $c \bar{q}$ pairs. The decay of $\bar{B} \rightarrow D^{* *} \ell^{-} \bar{v}_{\ell}$ where the $D^{*}$ meson decays to $D^{(*)} \pi^{0}$ peaks in the $m_{\text {miss }}^{2}$ distribution. These events are estimated using the Monte Carlo samples.

- Charge cross-feed events: these events come from the decay of $\bar{B} \rightarrow D^{* *}\left(\tau^{-} / \ell^{-}\right) \bar{v}_{\ell}$. These background events were reconstructed with the incorrect charge where one of the charges particles in the final state has been assigned to the different $B$ meson.

- Other $B \bar{B}$ background: These events come from the decay of $B \rightarrow D^{(*, *)} D_{s}^{(*, *)+}$ due to the large leptonic and semileptonic branching fractions of $D_{s}^{+}$mesons. We estimate these events using Monte Carlo sample and its contribution is fixed in the fitting process.

- Continuum background: these events come from the decay of $e^{+} e^{-} \rightarrow q \bar{q}(\gamma)$ pairs where $q$ can be $u, d, s, c, \tau$. To estimate this background we use the additional data sample of $40 \mathrm{fb}^{-1}$, taken at energy $40 \mathrm{MeV}$ below the $\Upsilon(4 S)$ resonance.

Figure 2 shows the distributions of $m_{m i s s}^{2}$ and $p_{\ell}^{*}$ variables for the signal events $(6 \%)$, normalization events $(82 \%)$, $B \rightarrow D^{* *} \ell v(4 \%)$, and combinatoric and continuum background (8\%). All distributions are normalized to 1000 entries after all selections are applied.
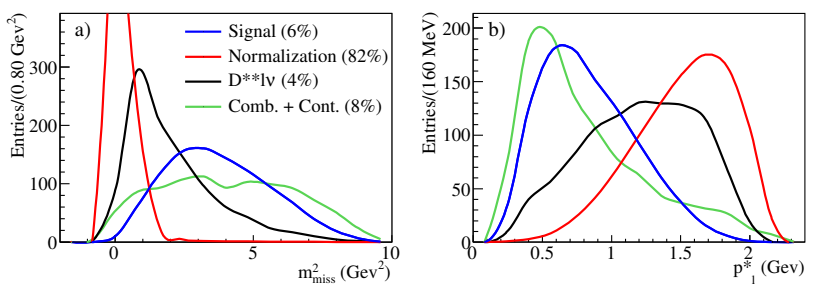

Figure 2. The comparison of the data and the fit projections for the four $D^{(*)} \ell$ samples: the signal events (6\%), normalization events (82\%), $B \rightarrow D^{* *} \ell v(4 \%)$, and combinatoric and continuum background (8\%). The $m_{E S}$ is on the left and the $p_{\ell}^{*}$ is on the right. All distributions are normalized to 1000 entries after all selections are applied.

The results of the signal and normalization yields are extracted using unbinned maximum-likelihood fit to the two dimensional, $m_{m i s s}^{2}-p_{\ell}^{*}$ contributions. The fit is performed simultaneously to the four $D^{(*)} \ell$ samples and four $D^{(*)} \pi^{0} \ell$ samples. The distributions of each $D^{(*)} \ell$ and $D^{(*)} \pi^{0} \ell$ sample is fitted to the sum of either eight or six contributions, respectively. The fit relies on $8 \times 4+6 \times 4=$ 56 probability density functions (PDFs). The two dimensional, $m_{m i s s}^{2}-p_{\ell}^{*}$ contributions for each of the 56 PDFs are 
described in detail using smooth non-parametric kernel estimator [13].

The $m_{m i s s}^{2}$ distributions of the signal events and the normalization events cane be easily distinguished due to the different number of neutrino as the undetected particles in its corresponding decays in the final state. However, the $m_{\text {miss }}^{2}$ distributions of the backgrounds resemble those of the signal events, and therefore in the fitting procedure these contributions are either fixed fitted or constrained by the $D^{(*)} \pi^{0} \ell$ Monte Carlo sample. Figure 3 shows the yield of $B \rightarrow D^{*} \tau v$ and the comparison of the $m_{m i s s}^{2}$ and $p_{\ell}^{*}$ distributions of the $B \rightarrow D^{*} \tau v$ (data points) with the projections of the results of the isospin-unconstrained fit. The region above the dashed line is the background component corresponds to $B \bar{B}$ background.

The region below the dashed line corresponds to the continuum background. Due to the charged cross-feed events the $m_{\text {miss }}^{2}$ distribution peak around $m_{\text {miss }}^{2}=0$ in the background component. However, in the $p_{\ell}^{*}$ distributions, we only include events with $m_{\text {miss }}^{2}>1 \mathrm{GeV}^{2}$.
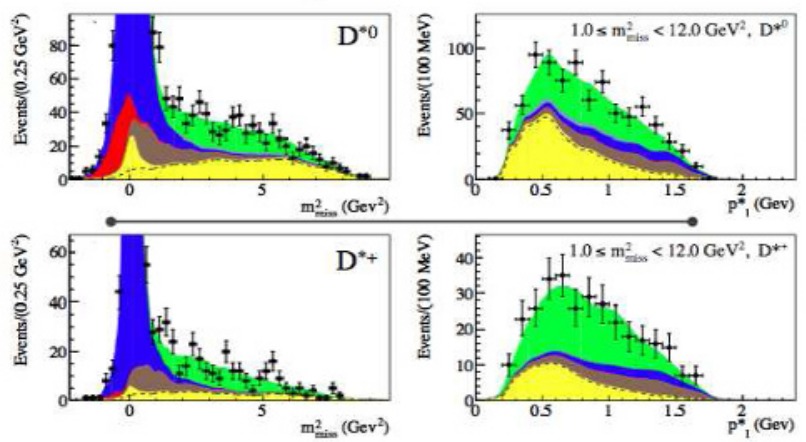

Figure 3. The comparison of the $m_{m i s s}^{2}$ (left) and $p_{\ell}^{*}$ (right) distributions of the $B \rightarrow D^{*} \tau v$ (data points) with the projections of the results of the isospin-unconstrained fit. The region above the dashed line is the background component corresponds to $B \bar{B}$ background. The region below the dashed line corresponds to the continuum background. In the $p_{\ell}^{*}$ distributions we only include events with $m_{\text {miss }}^{2}>1 \mathrm{GeV}^{2}$.

We extract the branching fraction ratios as define in the following

$$
\mathcal{R}\left(D^{(*)}\right)=\frac{N_{\text {sig }}}{N_{\text {norm }}} \times \frac{\epsilon_{\text {sig }}}{\epsilon_{\text {norm }}}
$$

where $N_{\text {sig }}$ and $N_{\text {norm }}$ are the number of signal and normalization events extracting from the fitting process, respectively. The $\epsilon_{\text {sig }} / \epsilon_{\text {norm }}$ is the ratio of the efficiencies of the signal and the normalization events. We impose the isospin relations of $\mathcal{R}\left(D^{*}\right) \equiv \mathcal{R}\left(D^{*+}\right)=\mathcal{R}\left(D^{* 0}\right)$ and $\mathcal{R}(D) \equiv \mathcal{R}\left(D^{+}\right)=\mathcal{R}\left(D^{0}\right)$. Table 1 shows the fit results of the yield of $B \rightarrow D^{*} \tau v$ with the statistical uncertainties only. Figure 4 shows the yield of $B \rightarrow D \tau v$ comparison of the $m_{m i s s}^{2}$ (left) and $p_{\ell}^{*}$ (right) distributions of the $B \rightarrow D \tau v$ (data points) with the projections of the results of the isospin-unconstrained fit.

The region above the dashed line is the background component corresponds to $B \bar{B}$ background. The region
Table 1. The yield results for the $B \rightarrow D^{*} \tau \nu$ channel where the uncertainties are the statistical only.

\begin{tabular}{llll}
\hline \hline Mode & $D^{* 0} \tau v$ & $D^{*+} \tau v$ & $D^{*} \tau v$ \\
\hline$N_{\text {sig }}$ & $639 \pm 62$ & $245 \pm 27$ & $888 \pm 63$ \\
$\mathcal{R}\left(D^{*}\right)$ & $0.32 \pm 0.03$ & $0.36 \pm 0.04$ & $0.33 \pm 0.02$ \\
$\mathcal{B}\left(D^{*} \tau v\right)$ & $1.71 \pm 0.17$ & $1.74 \pm 0.19$ & $1.76 \pm 0.13$ \\
\hline \hline
\end{tabular}

Table 2. The yield results for the $B \rightarrow D \tau v$ channel where the uncertainties are the statistical only.

\begin{tabular}{llll}
\hline \hline Mode & $D^{0} \tau v$ & $D^{+} \tau v$ & $D \tau \nu$ \\
\hline$N_{\text {sig }}$ & $314 \pm 60$ & $177 \pm 31$ & $489 \pm 63$ \\
$\mathcal{R}(D)$ & $0.43 \pm 0.08$ & $0.47 \pm 0.08$ & $0.44 \pm 0.06$ \\
$\mathcal{B}(D \tau v)$ & $0.99 \pm 0.19$ & $1.01 \pm 0.18$ & $1.02 \pm 0.13$ \\
\hline \hline
\end{tabular}

below the dashed line corresponds to the continuum background. In the $p_{\ell}^{*}$ distributions, we only include events with $m_{m i s s}^{2}>1 \mathrm{GeV}^{2}$. Table 2 shows the fit results of the
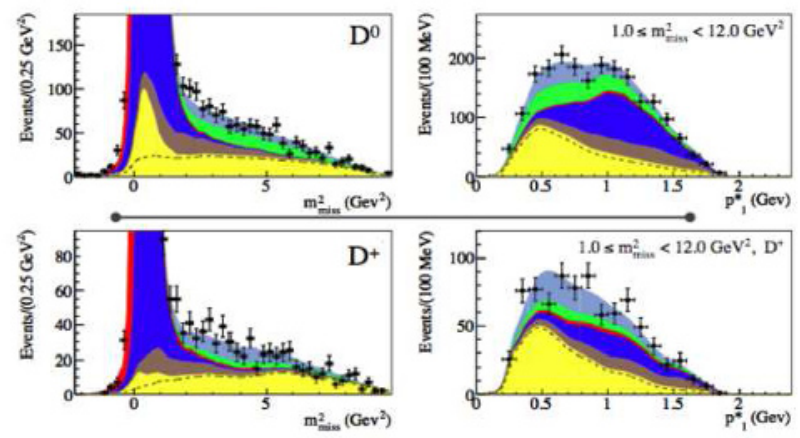

Figure 4. The comparison of the $m_{\text {miss }}^{2}$ (left) and $p_{\ell}^{*}$ (right) distributions of the $B \rightarrow D \tau v$ (data points). with the projections of the results of the isospin-unconstrained fit. The region above the dashed line is the background component corresponds to $B \bar{B}$ background. The region below the dashed line corresponds to the continuum background. In the $p_{\ell}^{*}$ distributions we only include events with $m_{\text {miss }}^{2}>1 \mathrm{GeV}^{2}$.

yield of $B \rightarrow D \tau v$ with the statistical uncertainties only.

\section{SYSTEMATICS UNCERTAINTIES}

The largest systematic uncertainties in this analysis is due to the poorly understood of the decay $B \rightarrow D^{* *}(\ell / \tau) v$ background. The systematic uncertainty due the PDF that describe these contributions including the uncertainty on the branching fractions of the four $B \rightarrow D^{* *} \ell v$ decays, the branching fraction ratio of $B \rightarrow D^{* *} \tau v$ to $B \rightarrow D^{* *} \ell v$, and its relative efficiency. We assign $2.1 \%$ on $\mathcal{R}(D)$ and $1.8 \%$ on $\mathcal{R}\left(D^{*}\right)$, respectively.

We also assign a systematic uncertainty due to the observed variation of the decay of $B \rightarrow D^{*} \eta \ell v$, nonresonance $B \rightarrow D^{*} \pi(\pi) \ell v, B \rightarrow D^{* *}(\ell / \tau) v$, and $D^{* *} \rightarrow$ $D^{(*)} \pi \pi$. They are $2.1 \%$ for $\mathcal{R}(D)$ and $2.6 \%$ for $\mathcal{R}\left(D^{*}\right)$.

The other largest systematic uncertainties are due to the continuum and $B \bar{B}$ backgrounds. We assign $4.9 \%$ for 
$\mathcal{R}(D)$ and $2.7 \%$ for $\mathcal{R}\left(D^{*}\right)$. The systematic uncertainties due to the PDFs for the signal and normalization decays are $4.3 \%$ for $\mathcal{R}(D)$ and $2.1 \%$ for $\mathcal{R}\left(D^{*}\right)$. The systematic uncertainties due to the efficiency ratios $\epsilon_{\text {sig }}$ and $\epsilon_{\text {norm }}$ are $2.6 \%$ on $\mathcal{R}(D)$ and $1.6 \%$ on $\mathcal{R}\left(D^{*}\right)$, respectively.

By choosing the decay of $\tau$ lepton only from the purely lepton decays: $\tau^{-} \rightarrow e^{-} \bar{v}_{e} \nu_{\tau}$ and $\tau^{-} \rightarrow e^{-} \bar{v}_{\mu} \nu_{\tau}$, uncertainties due to the particle identification, final state radiation, soft-pion reconstruction, and other related detector performance are largely cancel in taking the ratios. They only contribute about $1 \%$ in the systematic uncertainty.

All systematic uncertainties are added in quadrature to assign the total systematic uncertainty. There is a positive correlation between some of the systematic uncertainties $\mathcal{R}(D)$ and $\mathcal{R}\left(D^{*}\right)$. As a result the correlation of the total uncertainties is reduced to -0.27 for $\mathcal{R}(D)$ and $\mathcal{R}\left(D^{*}\right)$.

\section{CONCLUSIONS}

We have measured the $\mathcal{R}(D)=0.440 \pm 0.058 \pm 0.042$ and $\mathcal{R}\left(D^{*}\right)=0.332 \pm 0.024 \pm 0.018$. These ratios exceed the Standard Model predictions by $2.0 \sigma$ and $2.7 \sigma$, respectively. They are disagree with the SM prediction at the level of $3.4 \sigma$. The results are compatible with the results measured by the Belle Collaboration $[14,15]$. Together with the results measured by the Belle Collaboration, it could be an indication of new physics processes in $B$ mesons decay.

The measured values of $\mathcal{R}(D)$ and $\mathcal{R}\left(D^{*}\right)$ match the predictions of the particular Higgs model where the ratio of neutral Higgs field vacuum expectation values, $\tan \beta$, and the mass of the physical charged Higgs boson, $m_{H^{+}}$ is $\tan \beta / m_{H^{+}}=0.44 \pm 0.02 \mathrm{GeV}^{-1}$ and $\tan \beta / m_{H^{+}}=$ $0.75 \pm 0.04 \mathrm{GeV}^{-1}$, respectively. Figure 5 shows the comparison results of this paper with the charged Higgs boson of type II $2 \mathrm{HDM}$ predictions. The Standard Model predictions correspond to $\tan \beta / m_{H^{+}}=0$. However, these results

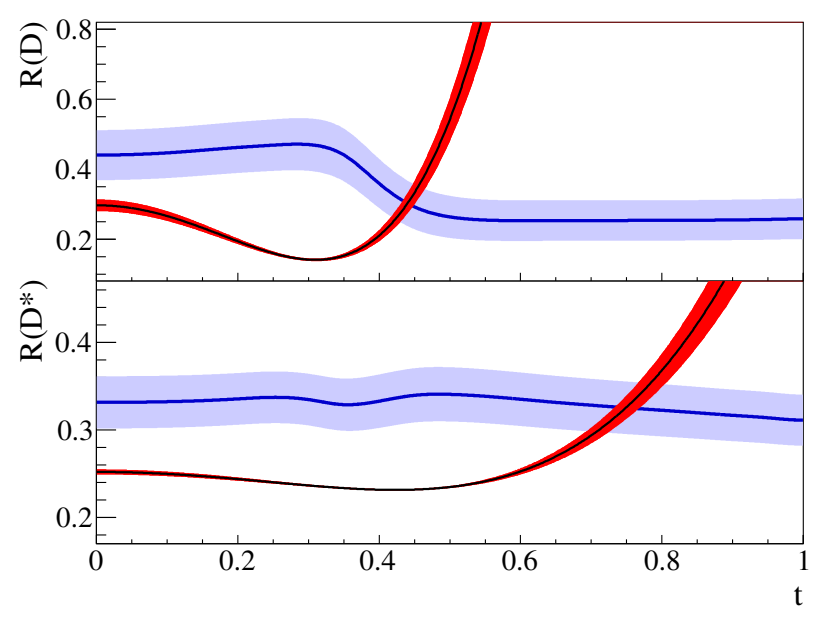

Figure 5. The comparison results of this paper (blue band) with predictions that include a charged Higgs boson of type II 2HDM (red band). The SM corresponds to $\tan \beta / m_{H^{+}}=0$. are not compatible with a charged Higgs boson in the type II $2 \mathrm{HDM}$ with a $99.8 \%$ confidence level for any value of $\tan \beta / m_{H^{+}}$. More general charged-Higgs models or other New Physics contributions may explain the access of $\mathcal{R}(D)$ and $\mathcal{R}\left(D^{*}\right)$.

\section{ACKNOWLEDGMENTS}

The author would like to thank the organizers of the Hadron Collider Physics Symposium 2012, Kyoto University, in Kyoto, Japan. The supports from the BABAR Collaboration, the University of South Alabama, and the University of Mississippi are gratefully acknowledged.

\section{References}

[1] P. Heiliger and L. Sehgal, Phys. Lett. B 229, 409 (1989).

[2] J. G. Korner and G. A. Schuler, Z. Phys. C 46, 93 (1990).

[3] D. S. Hwang and D. W. Kimm UEur. Phys. J. C. 14, 271 (2000).

[4] Particle Data Group, J. Beringer et al., Phys. Rev. D 86, 010001 (2012).

[5] B. Grzadkowski and W. Hou, Phys. Lett. B 283, 427 (1992).

[6] K. Kiers and A. Soni, Phys. Rev. D 56, 5786 (1997).

[7] M. Tanaka, Z. Phys. C 67, 321 (1995).

[8] U. Nierste, S. Trine, and S. Westhoff, Phys. Rev. D 78, 015006 (2008).

[9] J. F. Kamenik and F. Mescia, Phys. Rev. D 78, 014003 (2008).

[10] M. Tanaka and R. Watanabe, Phys. Rev. D 82, 034027 (2010).

[11] BABAR Collaboration, B. Aubert et al., Nucl. Instr. Methods Phys. Res., Sect. A 479, 1 (2002).

[12] S. Fajfer, J. F. Kamenik, and I. Nisandzic, Phys. Rev. D 85, 094025 (2012).

[13] K. S. Cranmer, Comp. Phys. Commun. 136, 198 (2001).

[14] Belle Collaboration, A. Matyja et al., Phys. Rev. Lett. 99, 191807 (2007).

[15] Belle Collaboration, A. Bozek et al., Phys. Rev. D 82, 072005 (2010). 